EUROPEAN JOURNAL OF PURE AND APPLIED MATHEMATICS

Vol. 13, No. 3, 2020, 579-586

ISSN 1307-5543 - www.ejpam.com

Published by New York Business Global

\title{
On solvability of $p$ - harmonic type equations in grand Sobolev spaces
}

\author{
Alik M. Najafov ${ }^{1,2, *}$, Sain T. Alekberli ${ }^{3}$ \\ 1 Azerbaijan University of Architecture and Construction, Baku, Azerbaijan \\ 2 Institute of Mathematics and Mechanics, National Academy of Science of Azerbaijan, \\ Baku, Azerbaijan \\ 3 Baku Engineering University, Baku, Azerbaijan
}

\begin{abstract}
In this paper with the help of variational method existence and uniqueness of solution of $p$ - harmonic type equations in grand Sobolev spaces is studied.

2020 Mathematics Subject Classifications: 35A01,35A02, 35A15, 35D30

Key Words and Phrases: $p$ - harmonic type equations, grand Sobolev space, variational method, Dirichlet problem
\end{abstract}

\section{Introduction and preliminary notes}

It is well known that the existence and uniqueness of Dirichlet problem for $p$-harmonic equations

$$
\begin{gathered}
\operatorname{div}\left(|\nabla u|^{p-2} \nabla u\right)=\operatorname{div} f, \\
\left.u\right|_{\partial G}=0
\end{gathered}
$$

in Sobolev and grand Sobolev spaces were studied, e.g., in [1, 2] see also [4-7, 10-13]. Namely, in these papers the different problems for $p$-harmonic equations were considered. Similar and various problems of partial differential equations in grand Sobolev, Besov and Morrey type spaces were studied in $[8,9,14-16,18-23]$ and others. Most of these papers were used the variational methods. Evidently, in the above-mentioned papers only $p$-harmonic equations (1) was considered.

In this paper we consider Dirichlet problem for $p$-harmonic type equation has a form

$$
\begin{gathered}
\operatorname{div}\left(|\nabla u|^{p-q} \nabla u\right)=\operatorname{div} f, \\
\left.u\right|_{\partial G}=\left.\varphi\right|_{\partial G},
\end{gathered}
$$

where $1<p<\infty ; \quad 2 \leq q<\infty ; \quad \varphi \in W_{p)}^{1}(G), f \in L_{(p-\varepsilon)^{\prime}}(G),(p-\varepsilon)^{\prime}=\frac{p-\varepsilon}{p-\varepsilon-1}$ and $G$ in $\mathbb{R}^{n}$ is a bounded domain.

*Corresponding author.

DOI: https://doi.org/10.29020/nybg.ejpam.v13i3.3762

Email addresses: aliknajafov@gmail.com (A.M. Najafov), sain.elekberli@bk.ru (S.T. Alekberli) 
Definition 1. ([6, 17, 23]) Denote by $W_{p)}^{1}(G)$ the grand Sobolev space of locally summable functions $u$ on $G$ having the weak partial derivatives $D_{x_{i}}^{1} u(i=1,2, \ldots, n)$ with the finite norm

$$
\|u\|_{W_{p)}^{1}(G)}=\|u\|_{L_{p)}(G)}+\|\nabla u\|_{L_{p)}(G)},
$$

where

$$
\|u\|_{L_{p)}(G)}=\sup _{0<\varepsilon<p-1}\left(\frac{\varepsilon}{|G|} \int_{G}|u(x)|^{p-\varepsilon} d x\right)^{\frac{1}{p-\varepsilon}}
$$

and $|G|$ is the Lebesgue measure of $G$.

We note that the correct choice of space for problem (3)-(4) is the grand Lebesgue space (or grand Sobolev space).

In this paper using the variational method an existence and uniqueness of solution to Dirichlet problem for $p$ - harmonic type equations (3)- (4) in grand Sobolev spaces is studied.

A weak solution for the problem (3)-(4) on $G$ is a function $u(x) \in W_{p)}^{1}(G)$, if $u-\varphi \in$ $\stackrel{\circ}{W_{p)}^{1}}(G)$ such that

$$
\sum_{i=1}^{n} \int_{G}|\nabla u|^{p-q} u_{x_{i}} \vartheta_{x_{i}} d x=\sum_{i=1}^{n} \int_{G} f \vartheta_{x_{i}} d x
$$

for every $\vartheta \in \stackrel{\circ}{W_{p}^{1}}(G)$.

\section{Main results}

In this section we prove the existence and uniqueness of weak solution (5) for the problem (3)-(4).

Theorem 1. Let $G \subset R^{n}$ is bounded domain, $1<p<\infty ; 2 \leq q<\infty ; g, h \in W_{p-(q-2)}^{1}(G)$, $\varphi \in W_{p)}^{1}(G)$ and $f \in L_{(p-\varepsilon)^{\prime}}^{1}$. Then the Dirichlet problem for pharmonic type equation (3) has a unique weak solutions in $W_{p)}^{1}(G)$.

Proof. Since functions $g$ and $h \in W_{p-(q-2)}^{1}(G)$, then we consider the bilinear functional as the form

$$
\begin{gathered}
F(g, h)=\sum_{i=1}^{n} \int_{G}|\nabla g|^{p-q} g_{x_{i}} h_{x_{i}} d x-\sum_{i=1}^{n} \int_{G} f h_{x_{i}} d x= \\
=I(g, h)-\sum_{i=1}^{n} \int_{G} f h_{x_{i}} d x=I(g, h)-(f, h),
\end{gathered}
$$


since $f \in L_{(p-\varepsilon)^{\prime}}(G),(p-\varepsilon)^{\prime}=\frac{p-\varepsilon}{p-\varepsilon-1}$. Consequently, we have

$$
\begin{gathered}
|I(g, g)|=|I(g)|=\left.\left|\sum_{i=1}^{n} \int_{G}\right| \nabla \mathrm{g}\right|^{p-q} g_{x_{i}} g_{x_{i}} d x \mid \leq \\
\leq \sum_{i=1}^{n} \int_{G}|\nabla \mathrm{g}|^{p-q} \\
\left|g_{x_{i}}\right|\left|g_{x_{i}}\right| d x=\sum_{i=1}^{n} \int_{G}|\nabla \mathrm{g}|^{p-q}\left|g_{x_{i}}\right|^{2} \quad d x= \\
=\int_{G}|\nabla \mathrm{g}|^{p-(q-2)} d x<\infty, \\
|I(g)| \leq\|\nabla \mathrm{g}\|_{L_{p-(q-2)}^{p-(q)}(G)}^{p .}
\end{gathered}
$$

Consequently, for every $q-2<\varepsilon<p-1$ function $g \in W_{p)}^{1}(G)$ and

$$
\|g\|_{W_{p)}^{1}(G)} \leq \mathrm{C}_{1}\|g\|_{W_{p-(q-2)}^{1}(G)},
$$

and, note that

$$
\|\nabla \mathrm{g}\|_{L_{p)}(G)}^{p-\varepsilon} \leq \mathrm{C}_{2}|I(g)|,
$$

where $C_{1}$ and $C_{2}$ are constants independents on function $g$.

The variational problem is stated as follows. Find a function $g \in W_{p)}^{1}(G)$ such that which gives the minimum value to the integral $F(g)$ and is unique. The Euler-Lagrange equation for the variational problem (6) under consideration is the equation (3). With the help of the inequality (7), we have

$$
\begin{gathered}
|F(g, g)|=|F(g)|=\left|I(g)-\sum_{i=1}^{n} \int_{G} f g_{x_{i}} d x\right| \geq|I(g)|- \\
-\left|\sum_{i=1}^{n} \int_{G} f g_{x_{i}} d x\right| \geq|I(g)|-\sum_{i=1}^{n}\left|\int_{G} f g_{x_{i}} d x\right| \geq|I(g)|-\left|\sum_{i=1}^{n} \int_{G} f g_{x_{i}} d x\right| \geq \\
\geq|I(g)|-\sum_{i=1}^{n} \int_{G}|f|\left|g_{x_{i}}\right| d x \geq C_{3}\|g\|_{W_{p)}^{1}(G)}^{p-\varepsilon}-\|g\|_{L_{p)}(G)}^{p-\varepsilon}- \\
-\|f\|_{L_{(p-\varepsilon)^{\prime}}(G)}\|\nabla g\|_{L_{p)}(G)} \geq C_{4}\|g\|_{W_{p)}^{1}(G)}=M_{0},
\end{gathered}
$$

$C_{3}$ and $C_{4}$ are constants independent on the function $g(x)$.

This means that $F(g)$ is lower bounded on $W_{p)}^{1}(G)$ show that there exists $g_{0} \in W_{p)}^{1}(G)$ such that $F\left(g_{0}\right)=\min _{g \in W_{p)}^{1}(G)} F(g)$. Fix some sequence $\left\{g_{m}\right\} \in W_{p)}^{1}(G)(m=1,2, \ldots)$ such that $\lim _{m \rightarrow \infty} F\left(g_{m}\right)=r_{0}$. Let $\sigma>0$ choose $m_{\sigma}$ so for $m \geq m_{\sigma}$ and $s=1,2, \ldots$ it holds $F\left(g_{m+s}\right)<r_{0}+\sigma$. Then noting that $\frac{1}{2}\left(g_{m+s}+g_{m}\right) \in W_{p)}^{1}(G)$ we have 
$F\left(\frac{g_{m+s}+g_{m}}{2}\right) \geq r_{0}$. By direct calculations we show that $I\left(\frac{g_{m+s}-g_{m}}{2}\right)<4 \sigma$, and we have $\left\|g_{m+s}+g_{m}\right\|_{W_{p)}^{1}(G)} \leq 2\left(\frac{\varepsilon}{C}\right)^{\frac{1}{p-\varepsilon}}$. This means that the sequence $\left\{g_{m}\right\}$ is fundamental in the spaces $W_{p)}^{1}(G)$, consequently in view of completeness the spaces $W_{p)}^{1}(G)$ there exist a function $g_{0} \in W_{p)}^{1}(G)$ such that $\lim _{m \rightarrow \infty}\left\|g_{m}-g_{0}\right\|_{W_{p)}^{1}(G)}=0$. By theorem on trace in $W_{p}^{1}(G),([3$, p.143]), we get

$$
W_{p)}^{1}(G) \rightarrow W_{p-\varepsilon}^{1}(G) \rightarrow L_{t-\varepsilon}\left(G_{k}\right), G_{k}=G \bigcap \mathbb{R}^{k}, p<t \leq \infty, 1 \leq k \leq n .
$$

So

$$
\left|F\left(g_{m}\right)-F\left(g_{0}\right)\right| \leq C\left\|g_{m}-g_{0}\right\|_{W_{p)}^{1}(G)}
$$

and hence it follows that $r_{0}=\lim _{m \rightarrow \infty} F\left(g_{m}\right)=F\left(g_{0}\right)$. Show that the function delivering minimum to the functional $F(g)$ is unique and satisfies equation $(3)$ in the space $W_{p)}^{1}(G)$. Since $g \in W_{p)}^{1}(G)$ and $F\left(g_{0}\right)=r_{0}$, we have

$$
\begin{gathered}
0 \leq I\left(\frac{g-g_{0}}{2}\right)=\frac{1}{2} F(g)+\frac{1}{2} F\left(g_{0}\right)-F\left(\frac{g+g_{0}}{2}\right) \leq \frac{r_{0}}{2}+\frac{r_{0}}{2}-r_{0}=0, \\
I\left(g-g_{0}\right)=0 .
\end{gathered}
$$

By $\left\|g_{m}-g_{0}\right\|_{W_{p)}^{1}(G)} \rightarrow 0, m \rightarrow \infty$, it follows that the function $g$ coincides with $g_{0}$ as an element of the space $W_{p)}^{1}(G)$. Again from the theorem on trace in space $W_{p)}^{1}(G)$, we have

$$
\left\|\left.\left(g_{m}-g_{0}\right)\right|_{\partial G}\right\|_{L_{t-\varepsilon}(\partial G)} \leq C\left\|g_{m}-g_{0}\right\|_{W_{p)}^{1}(G)} \rightarrow 0, \quad m \rightarrow \infty .
$$

Since

$$
\left\|\left.g_{m}\right|_{\partial G}-\left.\varphi\right|_{\partial G}\right\|_{L_{t-\varepsilon}(\partial G)} \rightarrow 0, m \rightarrow \infty
$$

therefore

$$
\left\|\left.g_{0}\right|_{\partial G}-\left.\varphi\right|_{\partial G}\right\|_{L_{t-\varepsilon}(\partial G)} \rightarrow 0 m \rightarrow \infty \text {. }
$$

Taking into account the condition $\frac{d}{d \mu}\left(F\left(g_{0}+\mu \omega\right)\right)_{\mu=0}=0$, show that the function $g_{0} \in W_{p)}^{1}(G)$, minimizing the integral $F(g)$ satisfies the following equation

$$
I\left(g_{0}, \omega\right)-(f, \omega)=0 .
$$

Now prove that the function $g_{0} \in W_{p)}^{1}(G)$ minimizing the integral $F(g)$ is the weak solution of the problem (3)-(4). By $\theta(t)$ we denote some monotonically decreasing function on the segment $\frac{1}{2} \leq t \leq 1$ and having the following properties

$$
\theta\left(\frac{1}{2}+0\right)=1, \theta(1-0)=-1, \theta^{(s)}\left(\frac{1}{2}+0\right)=\theta^{(s)}(1-0)=0, s=1,2, \ldots .
$$


The function

$$
\gamma(t)=\left\{\begin{array}{c}
\theta^{\prime}(t), \quad-\frac{1}{2} \leq t \leq 1 \\
0, \quad-\infty<t<\frac{1}{2}, 1<t<\infty
\end{array}\right.
$$

is infinitely differentiable and finite on the real line. Note that the function $\gamma$ satisfy condition

$$
\gamma^{(s)}\left(\frac{1}{2}+0\right)=\gamma(1-0),(s=1,2, \ldots) .
$$

Let $\delta>0$ and let $G_{\delta}=\left\{y: \rho\left(y, R^{n} \backslash G\right)>\delta\right\}$ be arbitrary point of the domain $G$, and $r=\rho\left(x, x_{0}\right)$. There $\rho\left(x, x_{0}\right)$ is the Euclidean distance between $x$ and $x_{0}$, where $x \in G$ and $x_{0}$ be a fixed point in $G$. Following Sobolev [24], we introduce the function

$$
\omega(x)=\gamma\left(\frac{r}{l_{1}}\right)-\gamma\left(\frac{r}{l_{2}}\right)
$$

for $0<l_{1}<l_{2}<\delta$. It is obvious that $\omega(x)$ is a infinitely differentiable finite function with a support lying on a annular domain $\frac{l_{1}}{2}<r<l_{2}$. Therefore $\omega \in C_{0}^{\infty}(G)$ and $\left.D^{(s)} \omega\right|_{\partial G}=0$ for all $s=1,2, \ldots$. Then from (8) by definition of the weak derivative it follows that

$$
\int_{G} K\left(\frac{r}{l_{1}}\right) g(x) d x=\int_{G} K\left(\frac{r}{l_{2}}\right) g(x) d x
$$

where

$$
K\left(\frac{r}{l_{i}}\right)=\operatorname{div}\left(\left|\nabla \gamma\left(\frac{r}{l_{i}}\right)\right|^{p-q} \nabla \gamma\left(\frac{r}{l_{i}}\right)\right)-\operatorname{div} f, \quad i=1,2 .
$$

Note that the function $K\left(\frac{r}{l_{i}}\right)$ having all properties of kernel. Namely, the following properties hold:

1) $K$ is infinitely differentiable function with support in the ball $r \leq l_{i}$;

2) The function $K$ and all its derivatives on sphere $R=h$ are zero;

3)

$$
\frac{1}{\tau_{n} l_{i}^{n}} \int_{G} K\left(\frac{r}{l_{i}}\right) d x=1
$$

where

$$
\tau_{n}=\frac{2 \pi^{\frac{n}{2}}}{\Gamma\left(\frac{n}{2}\right)} \int_{0}^{1} \xi^{n-1} K(\xi) d \xi
$$

Then for the function $g_{0}(x)$ we can constructed Sobolev's averaging $g_{0, l_{i}}(x), i=1,2$ on the ball $l_{i}(i=1,2)$ with centered at the point $x$ as

$$
g_{0, l_{i}}(x)=\frac{1}{\tau_{n} l_{i}^{n}} \int_{R^{n}} K\left(\frac{|z-x|}{l_{i}}\right) g_{0}(z) d z, \quad i=1,2 .
$$

The we can rewrite equality (9) in the form $g_{0, l_{1}}(x)=g_{0, l_{2}}(x)$. Consequently, for $l<\delta$

$$
g_{0, l}(x)=g_{0}(x) .
$$


Since the average functions $g_{0, l_{i}}(x), i=1,2$ are continuous and has continuous derivatives for any order, then $g_{0}(x)$ also is a kernel. Integrating by parts in the equality $I\left(g_{0}, \omega\right)-$ $(f, \omega)=0$, whence is the limit case

$$
\sum_{i=1}^{n} \int_{G} \omega(x) \frac{\partial}{\partial x_{i}}\left(\left|\nabla g_{0}\right|^{p-q} \frac{\partial}{\partial x_{i}} g_{0}(x)\right) d x=\sum_{i=1}^{n} \int_{G} \omega(x) \frac{\partial}{\partial x_{i}} f(x) d x
$$

Hence by the arbitrariness of the functions $\omega(x)$ it follows that

$$
\sum_{i=1}^{n} \frac{\partial}{\partial x_{i}}\left(\left|\nabla g_{0}\right|^{p-q} \frac{\partial}{\partial x_{i}} g_{0}(x)\right)=\sum_{i=1}^{n} \frac{\partial}{\partial x_{i}} f(x)
$$

i.e

$$
\operatorname{div}\left(\left|\nabla g_{0}\right|^{p-q} \nabla g_{0}\right)=\operatorname{divf} .
$$

Thus, solution of the variational problem (5) from the class $W_{p)}^{1}(G)$ is also solution of Dirichlet problem (3)-(4) and this solution is unique.

\section{Conclusion}

In conclusion, we note that for a $p$-harmonic type equation in the grand Sobolev space, a result is obtained on the existence and uniqueness of a weak solution.

\section{References}

[1] G Afrouzi and A Hadjian. Non Trivial Solutions for $p$ - Harmonic type Equations via a local minimum theorem for functionals. Taiwaness Journal of Math., 19(6):1731$1742,2015$.

[2] G Aronsson and P Lingvist. On $p$ - Harmonic Functions in the Plane and their Stream Functions. Jour. of Diff. Equations, 74:157-188, 1988.

[3] O V Besov, V P Ilyin, and S M Nikolskii. Integral Representations Functions and Embeddind Theorems. M. Nauka, Moscow, 1996.

[4] G Boccardo. Non linear elliptic and parabolic equations involving measure data. Jour. Func. Anal., 87:149-169, 1989.

[5] Y Deng and H Pi. Multiple solutions for $p$ - harmonic type equations. Nonlinear Anal., 71:4952-4959, 2009.

[6] A Fiorenza, M R Formica, and A Gogatishvili. On grand and small Lebesgue and Sobolev spaces and some applcations to PDEs. Diff. Equat. and Applic., 10(1):21-46, 2018. 
[7] A Fiorenza and C Sbordone. Existence and uniqueness results for solutions of nonlinear equations with right hand side in $L^{1}$. Stud. Math., 127(3):4959-4969, 1998.

[8] S Gala, Q Liu, and M A Ragusa. A new regularity criterion for the nematic liquid crystal flows. Applicable Analysis, 91(9):1741-1747, 2012.

[9] S Gala and M A Ragusa. Logarithmically improved regularity criterion for the Boussinesq equations in Besov spaces with negative indices. Applicable Analysis, 95(6):12711279, 2016.

[10] L Greco, T Iwaniec, and C Sbordone. Inverting the $p$ - harmonic operator. Manuscripta Math., 92(2):249-258, 1997.

[11] L Greco and A Verde. A reguliraty property of p- harmonic functions. Annal. Academ. Scien. Fennicae Math., 25:317-323, 2000.

[12] H Luiro and M Parviainen. Gradient walks and p- harmonic functions. Proc. Amer. of the Math. Soc., 145:4313-4324, 2017.

[13] J Manfredi. P- harmonic functions in the plane. Proc. of the Amer. Math. Soc., 103(2):473-479, 1988.

[14] A M Najafov. Problem on smoothness of solution of one class of hypoelliptic equations. Proc. A. Razm. Math. Inst., 140:131-139, 2006.

[15] A M Najafov. The Differential Properties of Functions from Sobolev-Morrey type Spaces of Fractional Order. Jour. Math. Res., 7(3):1-10, 2015.

[16] A M Najafov and A T Orujova. On the Solution of a Class of Partial Differential Equations. Electron. Jour. Qual. Theory Diff. Equ., 2017(44):1-9, 2017.

[17] A M Najafov and N R Rustamova. Some Differential Properties of Anisotropic Grand Sobolev-Morrey spaces. Trans. A. Razm. Math. Inst., 172:82-89, 2018.

[18] A M Najafov, N R Rustamova, and S T Alekberli. On Solvability of a Quasi-Elliptic Partial Differential Equations. Jour. of Ellip. and Parab. Equ., 2019(5):175187, 2019.

[19] N S Papageorgiou and A Scapellato. Nonlinear Robin problems with general potential and crossing reaction. Rend. Lincei-Mat. Appl., 30:1-29, 2019.

[20] N S Papageorgiou and A Scapellato. Concave-Convex Problems for the Robin pLaplacian Plus an Indefinite Potential. Mathematics, 8(3,421):1-27, 2020.

[21] S Polidoro and M A Ragusa. Harnack Inequality for Hypoelliptic Ultraparabolic Equations with a Singular Lower Order Term. Revista Matematica Iberoamericana, 24(3):1011-1046, 2008.

[22] M A Ragusa and A Tachikawa. Regularity for Minimizers for Functionals of Double Phase with Variable Exponents. Advances in Nonlinear Analysis, 9(1):710-728, 2020. 
[23] C Sbordone. Grand Sobolev Spaces and their Applications to Variational Problems. Le Matematiche (Catania), 51(2):335-347, 1996.

[24] S L Sobolev. Some Applications of Functional Analysis in Mathematical Physies. Novosibirsk, Russian, 1950. 